UCI-HEP-TR-2010-18

\title{
Gamma Ray Line Constraints on Effective Theories of Dark Matter
}

\author{
Jessica Goodman ${ }^{a}$, Masahiro Ibe $^{a}$, Arvind Rajaraman ${ }^{a}$, \\ William Shepherd ${ }^{a}$, Tim M.P. Tait ${ }^{a}$, and Hai-Bo Yu ${ }^{a, b}$ \\ a Department of Physics \& Astronomy, \\ University of California, Irvine, CA 92697 and \\ ${ }^{b}$ Michigan Center for Theoretical Physics, Department of Physics, \\ University of Michigan, Ann Arbor, MI 48109
}

(Dated: August 31, 2010)

\begin{abstract}
A monochromatic gamma ray line results when dark matter particles in the galactic halo annihilate to produce a two body final state which includes a photon. Such a signal is very distinctive from astrophysical backgrounds, and thus represents an incisive probe of theories of dark matter. We compare the recent null results of searches for gamma ray lines in the galactic center and other regions of the sky with the predictions of effective theories describing the interactions of dark matter particles with the Standard Model. We find that the null results of these searches provide constraints on the nature of dark matter interactions with ordinary matter which are complementary to constraints from other observables, and stronger than collider constraints in some cases.

PACS numbers: 95.35.+d, 14.80.-j
\end{abstract}




\section{INTRODUCTION}

The nature and identity of dark matter (DM) is one of the most significant outstanding problems in particle physics today. While cosmological measurements provide very compelling evidence for the existence of dark matter, basic details such as the mass of the dark matter particles and the nature of their interactions with ordinary matter have remained elusive. Nevertheless, it is widely believed that dark matter is a weakly interacting massive particle (WIMP). WIMPs are highly motivated by the many models of physics beyond the Standard Model (SM) which naturally produce stable candidates with the right properties and by the fact that the relic density of dark matter can be explained as a natural consequence of the thermal history of the Universe in such a scenario.

On the experimental side, if the dark matter has significant interactions with the Standard Model particles, it may be detectable at direct detection experiments which search for signals of the dark matter particle scattering off a nucleus. In recent years, several have reported anomalous results that are consistent with a light dark matter particle with mass of order $10 \mathrm{GeV}$ [1 3] (however, see [4 6] as well), leading to a surge of interest in the possibility of light dark matter [7-15]. Another experimental probe which is particularly effective for light dark matter is to look for signals of dark matter production at colliders. WIMP dark matter particles which couple to quarks and gluons can be pair produced along with SM radiation from the SM initial state, leading to a signal involving a high energy standard particle (such as a jet of hadrons or a photon) recoiling against missing momentum from the undetected WIMPs [16 20]. Colliders offer the advantage that the initial state and predicted backgrounds are under exquisite control, which makes the interpretations for given theories of WIMPs relatively straightforward.

A third broad class of experimental probes looks for the products of dark matter annihilating in the halo of our galaxy. The annihilation of WIMPs can produce distinctive and/or high energy SM particles which may stick out over the expected background from astrophysical sources. Such processes have the advantage that they search for the actual primordial dark matter particles in the cosmos, but this is also a disadvantage in that the rate from a given part of the sky depends on the amount of dark matter (squared) along the line of sight, which is not very well known. An even more serious challenge to searches of this type is the fact that in many cases the astrophysics is not very well understood, and 
thus the expected background is difficult to quantify.

Distinguishing a signal of WIMP annihilation from astrophysics is much easier if there are multiple handles defining the signal and/or features which even the uncertain background has difficulty producing. From both points of view, WIMP annihilations producing monochromatic lines in the spectrum of high energy gamma rays are a promising avenue [21 25]. Gamma rays have the advantage that they have a mean free path in the galaxy which is long enough that they generally point back to their sources. Thus, in searching for WIMP annihilations, the origin and morphology of a given signal can be compared with expectations for the distribution of dark matter and background sources. In addition, high energy photons are easy to detect, with the Fermi LAT satellite rapidly improving our understanding of celestial gamma rays with energies ranging from 100's of MeV to TeV.

Dark matter annihilations into SM final states generically produce gamma rays, which may be either radiated from charged final state particles or result from $\pi^{0}$ decays originating from the hadronization of colored final state particles. These "continuum" photons have an energy spectrum which cuts off at the mass of the WIMP. However, because the photons are dominantly produced as secondary (or tertiary) particles in the annihilation, the spectrum is highly peaked at low energies, and the presence of the cut-off may be difficult to ascertain in the data. A far more striking signature arises from gamma ray lines, which result when WIMPs annihilate into a two particle final state including a photon. Denoting the WIMPs as $\chi$, the reaction $\chi \chi \rightarrow \gamma X$ produces a photon with energy,

$$
E_{\gamma}=m_{\chi}\left(1-\frac{M_{X}^{2}}{4 m_{\chi}^{2}}\right) .
$$

At large enough energies, it would be difficult to imagine such a line resulting from any conventional astrophysical source. Since WIMPs are forbidden by observations from having large couplings to the photon, line emission processes are usually induced at loop level in perturbation theory. Thus, their rates are expected to be highly suppressed compared to the continuum photons. Nonetheless, the background rejection afforded by a monochromatic line signal easily compensates for the reduction in the expected rates. In this article, we will explore the null results of searches for gamma ray lines by the Fermi LAT to constrain the properties of dark matter interactions with the Standard Model.

If we are to assemble a coherent picture of what the inputs from direct detection, collider searches, and indirect detection experiments imply about the nature of dark matter, we need 
a framework which specifies how the WIMPs couple to various Standard Model fields. One popular way to proceed is to focus on a particular (usually pre-existing) model containing a WIMP candidate, e.g. a supersymmetric theory with $R$-parity conservation or a theory with Universal Extra Dimensions. Such explorations are interesting, and probe motivated visions for physics beyond the Standard Model. However, they are often not generic. Thus, rather than focus on a specific model, we choose to use the powerful machinery of effective field theory (EFT) to capture features from a broad class of WMP models. We specialize to the class of EFTs in which the relevant degrees of freedom are the dark matter particle itself and the Standard Model - a theory with a "Maverick" WIMP [18].

A Maverick WIMP couples to the Standard Model through high dimensional operators. The precise form of the interactions will depend on whether the dark matter is a fermion or a scalar. These operators only accurately represent the physics in the regime where the EFT correctly captures all of the relevant physics (at energies lower than the lightest degree of freedom after the dark matter itself). Provided this is true for all experiments of interest, the EFT provides a common language which allows one to compare the constraints from different types of experiments. This approach has previously been fruitful in exploring the results of direct detection [17, 26], collider searches [17-20, 27], and continuum gamma ray production from WIMP annihilation [17, 28]. Here we extend the analysis to include indirect searches for gamma ray lines.

We begin in the next section by defining the EFTs and enumerating the set of operators we consider. We simplify our analysis by considering WIMPs which are singlets under SM gauge transformations; the extension to other cases is straight-forward. We proceed by finding the annihilation rate into two body final states including photons (which typically involve a one loop Feynman diagram). Given our EFT assumptions, the allowed final states include $\gamma \gamma, \gamma Z$, or $\gamma h^{0}$. Since the SM Higgs has yet to be observed, and thus its mass is unknown, we restrict our attention to the $\gamma \gamma$ and $\gamma Z$ cases. We compare these rates to the bounds on gamma ray line searches from Fermi, and find corresponding bounds on the dark matter interactions. We also present the comparison to current bounds coming from colliders and direct detection methods and finish with a discussion of our results. 


\section{EFFECTIVE THEORY OF WIMP INTERACTIONS}

Following [19], we assume that the WIMP $\chi$ is the only relevant new particle in the energy range of interest, and thus the EFT contains the Standard Model and $\chi$. We restrict our attention to the cases where $\chi$ is a SM gauge singlet, but allow it to be a real scalar, a complex scalar, a Majorana fermion, or a Dirac fermion. We consider each of these spin cases separately, and we ignore spin one or higher Lorentz representations.

Since the WIMP is neutral under the SM, the only way it can communicate with the Standard Model fields is through higher dimensional operators ${ }^{1}$ which factor into a WIMP term multiplied by product of SM fields which itself forms a gauge singlet. The two factors are contracted together to form an over-all Lorentz invariant. We are particularly interested in operators which couple the WIMP to quarks and gluons, since these operators can produce signals at direct detection experiments.

The WIMP factors depend sensitively on the spin of $\chi$. For a real scalar, only the factor $\chi^{2}$ is non-vanishing; the $\chi \partial_{\mu} \chi$ construction can be integrated by parts and moved entirely to the SM term. For a complex scalar, $\chi^{\dagger} \chi$ and $\chi^{\dagger} \partial_{\mu} \chi$ are both independent. Terms with more than one derivative acting on $\chi$ are always subleading in the EFT. For a fermion $\chi$, the leading operators are of the form $\bar{\chi} \Gamma \chi$, where the matrices $\Gamma$ are one of the set of $4 \times 4$ matrices, a complete basis for which is,

$$
\left\{1, \gamma^{5}, \gamma^{\mu}, \gamma^{\mu} \gamma^{5}, \sigma^{\mu \nu}\right\}
$$

Note that $\gamma^{\mu}$ and $\sigma^{\mu \nu}$ vanish for a Majorana $\chi$. Terms with derivatives acting on a fermion WIMP are also subleading in the EFT. In constructing the interactions for a Dirac fermion or complex scalar WIMP, we restrict ourselves to those terms which leave a $U(1)_{\chi}$ symmetry unbroken, as the lack of this symmetry would also allow for mass terms which would split the single Dirac WIMP into two Majorana fermions or the complex scalar WIMP into two real scalars.

The Standard Model factors consist of quark operators $\bar{q} \Gamma q$ (with the color indices of the quarks contracted), where $\Gamma$ are matrices from the same set as in Equation (2), including:

\footnotetext{
${ }^{1}$ We assume that the Higgs is heavy enough to be integrated out of the EFT, in which case it may be responsible for UV-completing some of the operators which we do consider.
} 
- Quark scalar terms are constructed as

$$
\sum_{q} m_{q} \bar{q} q \quad \text { or } \quad \sum_{q} m_{q} \bar{q} \gamma_{5} q .
$$

- Quark vector terms are constructed:

$$
\sum_{q} \bar{q} \gamma_{\mu} q \quad \text { or } \quad \sum_{q} \bar{q} \gamma_{\mu} \gamma_{5} q
$$

- Quark tensor terms are constructed:

$$
\sum_{q} \bar{q} \sigma_{\mu \nu} q
$$

The constructions for scalar and vector bilinears may be further motivated by minimal flavor violation [29]. The normalization of the tensor bilinear facilitates comparison with direct detection rates. One could also write down analogous couplings to leptons. In the case of the mass-suppressed operators this would have only a small effect because leptons are light compared to quarks. In the cases where the mass of the SM particle does not appear in the coupling the presence of a coupling to leptons with identical strength would enhance the gamma ray line cross section by a factor of $64 / 25$, but such operators have very suppressed contributions to direct detection, and so we do not consider them here. At one higher dimension, we also have dark matter couplings to $G_{\mu \nu}^{a} G_{\alpha \beta}^{a}$, where $G_{\mu \nu}^{a}$ is the color field strength, and the Lorentz indices are contracted in all possible ways. The gluonic operators are normalized by $\alpha_{s}$, which captures the dominant renormalization group running and is motivated by the fact that such interactions are most likely generated at the loop level by integrating out a set of massive colored states.

The coefficient of each operator is a dimensionful parameter, expressed in terms of the appropriate power of the suppression scale $M_{*}$, which has dimensions of [energy]. Each operator has its own $M_{*}$, but since we will consider one operator at a time (which need not be the actual case but nonetheless captures the physics when one operator dominates a given process) we refer to the set of parameters collectively as $M_{*}$.

In the case of a Dirac WIMP, another possibility is a direct coupling of the dark matter to photons through either an electric or magnetic dipole interaction

$$
D \bar{\chi} \sigma_{\mu \nu} \gamma_{5} \chi F^{\mu \nu} \quad \text { or } \quad M \bar{\chi} \sigma_{\mu \nu} \chi F^{\mu \nu}
$$




\begin{tabular}{|c|c|c|}
\hline Name & Operator & Coefficient \\
\hline D1 & $\bar{\chi} \chi \bar{q} q$ & $m_{q} / M_{*}^{3}$ \\
D2 & $\bar{\chi} \gamma^{5} \chi \bar{q} q$ & $i m_{q} / M_{*}^{3}$ \\
D3 & $\bar{\chi} \chi \bar{q} \gamma^{5} q$ & $i m_{q} / M_{*}^{3}$ \\
D4 & $\bar{\chi} \gamma^{5} \chi \bar{q} \gamma^{5} q$ & $m_{q} / M_{*}^{3}$ \\
D5 & $\bar{\chi} \gamma^{\mu} \chi \bar{q} \gamma_{\mu} q$ & $1 / M_{*}^{2}$ \\
D6 & $\bar{\chi} \gamma^{\mu} \gamma^{5} \chi \bar{q} \gamma_{\mu} q$ & $1 / M_{*}^{2}$ \\
D7 & $\bar{\chi} \gamma^{\mu} \chi \bar{q} \gamma_{\mu} \gamma^{5} q$ & $1 / M_{*}^{2}$ \\
D8 & $\bar{\chi} \gamma^{\mu} \gamma^{5} \chi \bar{q} \gamma_{\mu} \gamma^{5} q$ & $1 / M_{*}^{2}$ \\
D9 & $\bar{\chi} \sigma^{\mu \nu} \chi \bar{q} \sigma_{\mu \nu} q$ & $1 / M_{*}^{2}$ \\
D10 & $\bar{\chi} \sigma_{\mu \nu} \gamma^{5} \chi \bar{q} \sigma_{\mu \nu} q$ & $i / M_{*}^{2}$ \\
D11 & $\bar{\chi} \chi G_{\mu \nu} G^{\mu \nu}$ & $\alpha_{s} / 4 M_{*}^{3}$ \\
D12 & $\bar{\chi} \gamma^{5} \chi G_{\mu \nu} G^{\mu \nu}$ & $i \alpha_{s} / 4 M_{*}^{3}$ \\
D13 & $\bar{\chi} \chi G_{\mu \nu} \tilde{G}^{\mu \nu}$ & $i \alpha_{s} / 4 M_{*}^{3}$ \\
D14 & $\bar{\chi} \gamma^{5} \chi G_{\mu \nu} \tilde{G}^{\mu \nu}$ & $\alpha_{s} / 4 M_{*}^{3}$ \\
D15 & $\bar{\chi} \sigma^{\mu \nu} \chi F_{\mu \nu}$ & $M$ \\
D16 & $\bar{\chi} \sigma_{\mu \nu} \gamma^{5} \chi F_{\mu \nu}$ & $D$ \\
\hline M1 & $\bar{\chi} \chi \bar{q} q$ & $m_{q} / 2 M_{*}^{3}$ \\
M2 & $\bar{\chi} \gamma^{5} \chi \bar{q} q$ & $i m_{q} / 2 M_{*}^{3}$ \\
\hline
\end{tabular}

\begin{tabular}{|c|c|c|}
\hline Name & Operator & Coefficient \\
\hline M3 & $\bar{\chi} \chi \bar{q} \gamma^{5} q$ & $i m_{q} / 2 M_{*}^{3}$ \\
M4 & $\bar{\chi} \gamma^{5} \chi \bar{q} \gamma^{5} q$ & $m_{q} / 2 M_{*}^{3}$ \\
M5 & $\bar{\chi} \gamma^{\mu} \gamma^{5} \chi \bar{q} \gamma_{\mu} q$ & $1 / 2 M_{*}^{2}$ \\
$\mathrm{M} 6$ & $\bar{\chi} \gamma^{\mu} \gamma^{5} \chi \bar{q} \gamma_{\mu} \gamma^{5} q$ & $1 / 2 M_{*}^{2}$ \\
$\mathrm{M} 7$ & $\bar{\chi} \chi G_{\mu \nu} G^{\mu \nu}$ & $\alpha_{s} / 8 M_{*}^{3}$ \\
$\mathrm{M} 8$ & $\bar{\chi} \gamma^{5} \chi G_{\mu \nu} G^{\mu \nu}$ & $i \alpha_{s} / 8 M_{*}^{3}$ \\
$\mathrm{M} 9$ & $\bar{\chi} \chi G_{\mu \nu} \tilde{G}^{\mu \nu}$ & $i \alpha_{s} / 8 M_{*}^{3}$ \\
$\mathrm{M} 10$ & $\bar{\chi} \gamma^{5} \chi G_{\mu \nu} \tilde{G}^{\mu \nu}$ & $\alpha_{s} / 8 M_{*}^{3}$ \\
\hline $\mathrm{C} 1$ & $\chi^{\dagger} \chi \bar{q} q$ & $m_{q} / M_{*}^{2}$ \\
$\mathrm{C} 2$ & $\chi^{\dagger} \chi \bar{q} \gamma^{5} q$ & $i m_{q} / M_{*}^{2}$ \\
$\mathrm{C} 3$ & $\chi^{\dagger} \partial_{\mu} \chi \bar{q} \gamma^{\mu} q$ & $1 / M_{*}^{2}$ \\
$\mathrm{C} 4$ & $\chi^{\dagger} \partial_{\mu} \chi \bar{q} \gamma^{\mu} \gamma^{5} q$ & $1 / M_{*}^{2}$ \\
$\mathrm{C} 5$ & $\chi^{\dagger} \chi G_{\mu \nu} G^{\mu \nu}$ & $\alpha_{s} / 4 M_{*}^{2}$ \\
$\mathrm{C} 6$ & $\chi^{\dagger} \chi G_{\mu \nu} \tilde{G}^{\mu \nu}$ & $i \alpha_{s} / 4 M_{*}^{2}$ \\
\hline $\mathrm{R} 1$ & $\chi^{2} \bar{q} q$ & $m_{q} / 2 M_{*}^{2}$ \\
$\mathrm{R} 2$ & $\chi^{2} \bar{q} \gamma^{5} q$ & $i m_{q} / 2 M_{*}^{2}$ \\
$\mathrm{R} 3$ & $\chi^{2} G_{\mu \nu} G^{\mu \nu}$ & $\alpha_{s} / 8 M_{*}^{2}$ \\
$\mathrm{R} 4$ & $\chi^{2} G_{\mu \nu} \tilde{G}^{\mu \nu}$ & $i \alpha_{s} / 8 M_{*}^{2}$ \\
\hline
\end{tabular}

TABLE I: Operators coupling WIMPs to SM particles. The operator names beginning with D, M, C, R apply to WIMPs that are Dirac fermions, Majorana fermions, complex scalars or real scalars respectively.

where we refer to the coefficients of these two operators as $D$ and $M$, respectively. As we shall see, these operators are probed particularly well by searches for gamma ray lines due to the direct coupling of the dark matter to the photon [30 34]. We note that there has been recent interest in dark matter with dipole interactions, which have the potential to reconcile the DAMA signal while remaining consistent with the null search results from CDMS and XENON [35 39].

The complete list of operators that we consider is shown in Table I. We adopt a naming convention where the initial letter refers to the spin of $\chi$ : $\mathrm{D}$ for Dirac fermion, $\mathrm{M}$ for 


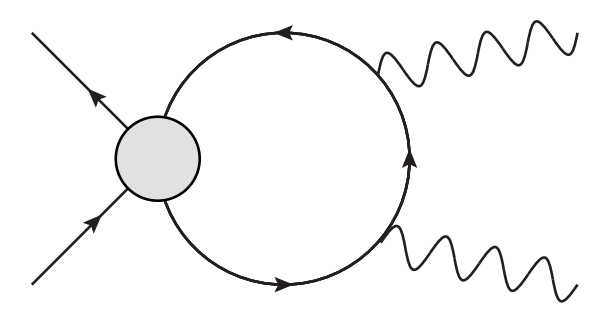

FIG. 1: Representative Feynman diagram for the loop level annihilation of two DM particles $\chi$ to a photon and a second vector boson, either another photon or a $Z$ boson, through an operator coupling the DM to SM quarks (represented as the shaded circle).

Majorana, C for complex scalar, and R for real scalar and the number specifies the particular operator belonging to a given WIMP spin. Within each family, the earlier numbers refer to coupling to quark scalar bilinears (D1-4, M1-4, C1-2, and R1-2), the middle numbers to quark vector bilinears (D5-8, M5-6, and C3-4) and quark tensor bilinears (D9-10) and the largest numbers to coupling to gluons (D11-14, M7-10, C5-6, and R3-4). The WIMP electric and magnetic dipole moment operators are labelled D15 and D16.

\section{GAMMA RAY LINE SEARCH CONSTRAINTS}

We compute the rate for the processes $\chi \chi \rightarrow \gamma \gamma$ and $\chi \chi \rightarrow \gamma Z$ for each of the operators considered above. Generally, stronger bounds arise from the $\gamma \gamma$ process because it produces two photons per annihilation (compensating for the $Z$ coupling to quarks being typically a little stronger than the photon). Consequently, we consider the $\gamma Z$ final state only in the case where annihilation into $\gamma \gamma$ vanishes. For the cases with a Dirac fermion or complex scalar, we assume that the dark matter in our galactic halo is composed of equal numbers of particles and anti-particles. It should be borne in mind that one could evade the constraints from any annihilation process if the interactions preserve the $U(1)_{\chi}$ symmetry and the galactic halo is made entirely of WIMPs or anti-WIMPs.

For the operators D15 and D16 mediating a direct interaction between the WIMPs and the photon, this process occurs at tree level. Generally, the quark operators mediate annihilations into $\gamma \gamma$ or $\gamma Z$ at the one loop level as shown in Figure 1. For the operators of the form $\bar{\chi} \Gamma^{\mu} \chi \bar{q} \gamma_{\mu} q$, a final state containing two photons is forbidden by the Landau-Yang

theorem [40]. For these operators, we rely on $\chi \chi \rightarrow \gamma Z$ to determine the implications of 
searches for gamma ray lines. For operators coupling the WIMPs directly to gluons and for the tensor operators D9 and D10, the leading contribution to $\gamma \gamma$ and $\gamma Z$ final states occurs at two loops, and as a result the rate is expected to be small enough that these operators are much better probed by collider searches [19]. Thus, we do not consider indirect bounds on these operators.

The one loop amplitudes were generated using the FeynArts package [41] from which cross sections were derived using FormCalc [42]. As a cross check, the results for the operators D2 and D4 were compared at the analytic level of the scalar loop integrals (after the PassarinoVeltman reduction [43] was performed by hand), and agreement found with the FeynArts results. Despite the fact that our EFT is nonrenormalizable, all of the one loop amplitudes for annihilation into $\gamma \gamma$ and $\gamma Z$ are finite, insensitive to the detailed UV theory.

Armed with the cross sections for WIMP annihilation into $\gamma \gamma$ or $\gamma Z$, we compare with the bounds from the Fermi LAT null searches for gamma ray lines. The Fermi LAT has released the results of searches from $30 \mathrm{GeV}$ to $200 \mathrm{GeV}$ [45], and is eventually expected to reach lower masses as well.

In terms of the annihilation cross section and WIMP mass, the flux produced in monochromatic lines is given by

$$
\Phi_{S}=\frac{1}{4 \pi} \frac{r_{\odot} \rho_{\odot}^{2}}{m_{\chi}^{2}}\langle\sigma(\chi \chi \rightarrow \gamma \gamma) v\rangle \bar{J} \times(\Delta \Omega)
$$

where $\Delta \Omega$ is the size of the angular acceptance and $\bar{J}$ encodes the integral of the dark matter density $\rho$ squared along the line of sight,

$$
\bar{J} \equiv \frac{1}{\Delta \Omega} \int_{\Delta \Omega} d \Omega \int_{\operatorname{LOS}} \frac{d l}{r_{\odot}} \frac{\rho^{2}(l, \Omega)}{\rho_{\odot}^{2}}
$$

with $r_{\odot}=8.5 \mathrm{kpc}$ the distance from the sun to the galactic center and $\rho_{\odot}$ the local density of dark matter. For annihilations into $\gamma Z$, the final state photon multiplicity is reduced by a factor of two, and the energy of the line is mapped onto the WIMP mass via Eq. (1) with $M_{X}=M_{Z}$.

The quantity $\bar{J}$ is typically derived from $N$-body simulations simulating the formation and evolution of Milky Way-like galaxies. However, the simulations cannot resolve scales of order the galactic center and do not include effects from baryonic matter which may strongly affect the dark matter profile close to the core. As a result, it represents the largest uncertainty in mapping the experimental results onto cross sections (and from there onto 
the coefficients of operators). Typical choices of the dark matter density thought to provide realistic pictures of the milky way halo are the Navarro-Frenk-White NFW [46] or "Einasto" [47] profiles. In order to derive the most conservative bounds, we choose the Fermi limits corresponding to an isothermal profile [48] which results in a modest $\bar{J}$ and correspondingly weaker limits (about a factor of two compared to NFW for the Fermi line search region of interest [45]) on the annihilation cross section.

The null results of searches for gamma ray lines, together with our choice of the isothermal dark matter distribution, puts a 95\% CL bound on on the cross section $\sigma(\chi \chi \rightarrow \gamma \gamma$ ) (or in some cases $\sigma(\chi \chi \rightarrow \gamma Z))$. These are mapped into a bound on the parameter $M_{*}$ which appears in the coefficients of each operator. These bounds ${ }^{2}$ are presented in Figures 5 -9, along with the region where each operator provides the correct thermal relic density and the previous $95 \%$ CL bounds from Tevatron data (as well as the future prospects for a $5 \sigma$ detection at the LHC) [19].

We find that for the cases of operators D1 and D3, the line searches from Fermi are actually stronger than collider bounds for WIMP masses greater than about $50 \mathrm{GeV}$ and for D2 and D4, are stronger than the Tevatron bounds for all WIMP masses covered by the Fermi line search and stronger than the LHC prospects for masses greater than about 100 GeV. For operators D5 and D7, Fermi bounds are stronger for masses greater than about $90 \mathrm{GeV}$. For operators $\mathrm{C} 1, \mathrm{C} 2$, and $\mathrm{C} 4$, the line searches are stronger than the Tevatron bounds for larger WIMP masses, with $\mathrm{C} 1$ and $\mathrm{C} 2$ much more strongly probed than is even possible at the LHC. Overall, our results show a rich potential for synergy between different types of experimental probes of dark matter.

For the cases where the dark matter couples directly to photons (operators D15 and D16), the existing bounds from Fermi are shown in Fig. 7 as the solid red line beyond masses of 30 $\mathrm{GeV}$. While the current Fermi limits only go down to lines of energy $30 \mathrm{GeV}$, the Fermi LAT is also potentially sensitive to much smaller energies. In the absence of a published study, we extrapolate the existing bounds of [45] to lower masses. While these extrapolations are hopefully semi-realistic at masses not much below the current lower limit of $30 \mathrm{GeV}$, we

\footnotetext{
${ }^{2}$ The bounds on Majorana and real scalar WIMPs are simply related to the corresponding bounds from Dirac and complex scalar WIMPs respectively, once one notes that the bounds on the identical particle cross sections are a factor of two better than the corresponding bounds on $\chi \bar{\chi}$ (particle-antiparticle) annihilation.
} 
caution that for very low masses they may be unrealistic and/or unconservative. Obviously, the most ideal situation would be to have updated limits by Fermi itself in the future. To estimate the background, we assume that the background is described by a power law below $30 \mathrm{GeV}$. The data [45] between 30 and $55 \mathrm{GeV}$ are described by a power law,

$$
\frac{d B}{d E}=1.22 \times 10^{6} \mathrm{GeV}^{1.39} \times E^{-2.39}
$$

where $d B / d E$ is the differential number of events (corresponding to an 11 month exposure) in energy. We treat the energy resolution of the LAT as $\Delta E / E=10 \%$, constant in energy. Choosing a bin size corresponding to twice the experimental resolution, we find the expected number of background events in the bin by integrating the background function, Eq. (9). We place $95 \%$ confidence level expected limits on a hypothesized signal line producing $S$ events by requiring $S / \sqrt{B}<1.96$. As a check of the consistency of our parameterization, we derive the flux bounds in [45] and find they are consistent with the actual experimental results to within better than $5 \%$ over the entire range $30-200 \mathrm{GeV}$. The resulting expected bounds on $M_{*}, D$, and $M$ from the extrapolated Fermi line search are plotted in Figures 5 - 9 as dashed lines extending below $30 \mathrm{GeV}$.

\section{DISCUSSION AND CONCLUSIONS}

The power of an effective theory description of WIMP interactions is that it also allows one to make predictions for any observable in the domain of validity of the effective theory. In Figure2, we show the parameter space for a complex scalar WIMP scattering through a spinindependent interaction with a nucleon along with some of the existing bounds and prospects for direct detection experiments [3, 5, 49]. The limits from gamma ray lines are worse than the current limits from Xenon 10 and CDMS, but are of the same order for WIMP masses around $200 \mathrm{GeV}$. If the LAT were to observe a gamma ray line signal in the near future, this would (for example) exclude these combinations of WIMP masses and couplings as being responsible. In Figure 3, we show the parameter space for a Dirac WIMP scattering through a spin-dependent interaction along with some of the relevant direct detection experiments [50]. We see that, as was also true for colliders, the line searches are stronger for some operators than near future prospects for spin-dependent scattering. Again, in the presence of a signal this would favor some WIMP spins and operators over others. 


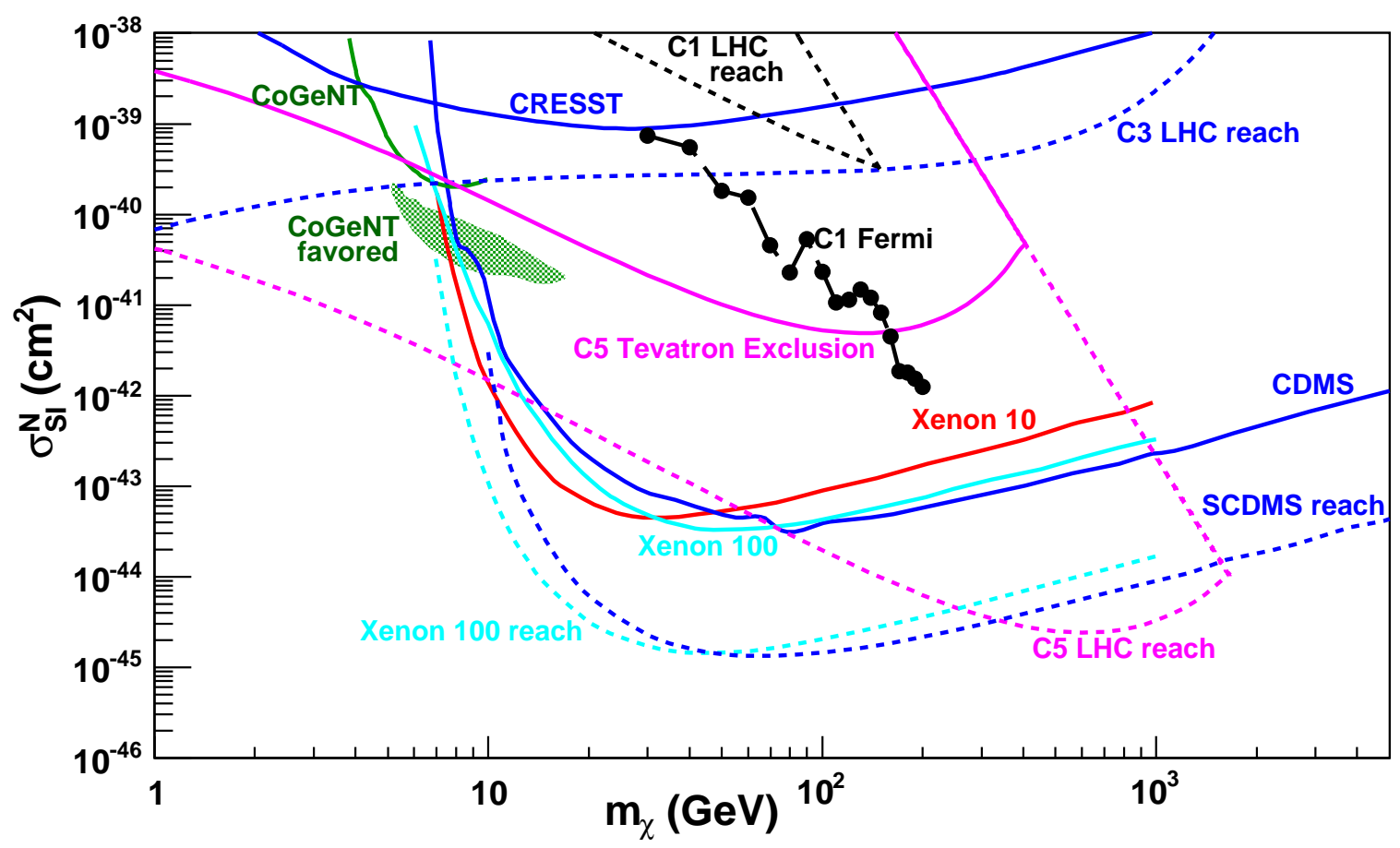

FIG. 2: Graph of the parameter space for spin-independent direct detection of WIMPs, in terms of the WIMP mass and cross section for scattering on a nucleon, including current bounds from direct detection, Tevatron data, and the Fermi line search (solid lines, as labelled), and projected lines for direct detection experiments and the LHC (dashed lines, as labelled). The bounds on complex scalars are shown. The bounds for the operator C3 are above the axes displayed here. Fermi bounds on real scalars are obtained by improving the corresponding bounds for the complex scalars by a factor of 2 . Fermi bounds on fermion operators are weak and outside the scale of this plot.

In Figure 4 we present the parameter space for a Majorana WIMP annihilating into two photons through the operator M6, in the parameter space of $m_{\chi}$ and $\sigma(\chi \chi \rightarrow \gamma \gamma)$. Together with the Fermi line search data, we have shown the best limits from direct detection experiments [50] and the Tevatron limits [19]. For M6, the Fermi line search is currently the best available limits for the masses to which they apply, with the Tevatron limits comparable and taking over at low WIMP masses. Direct detection limits become the most constraining for WIMP masses above $\gtrsim 300 \mathrm{GeV}$.

Also shown in Figure 4 is the parameter space for which "Magnetic inelastic Dark Matter" 


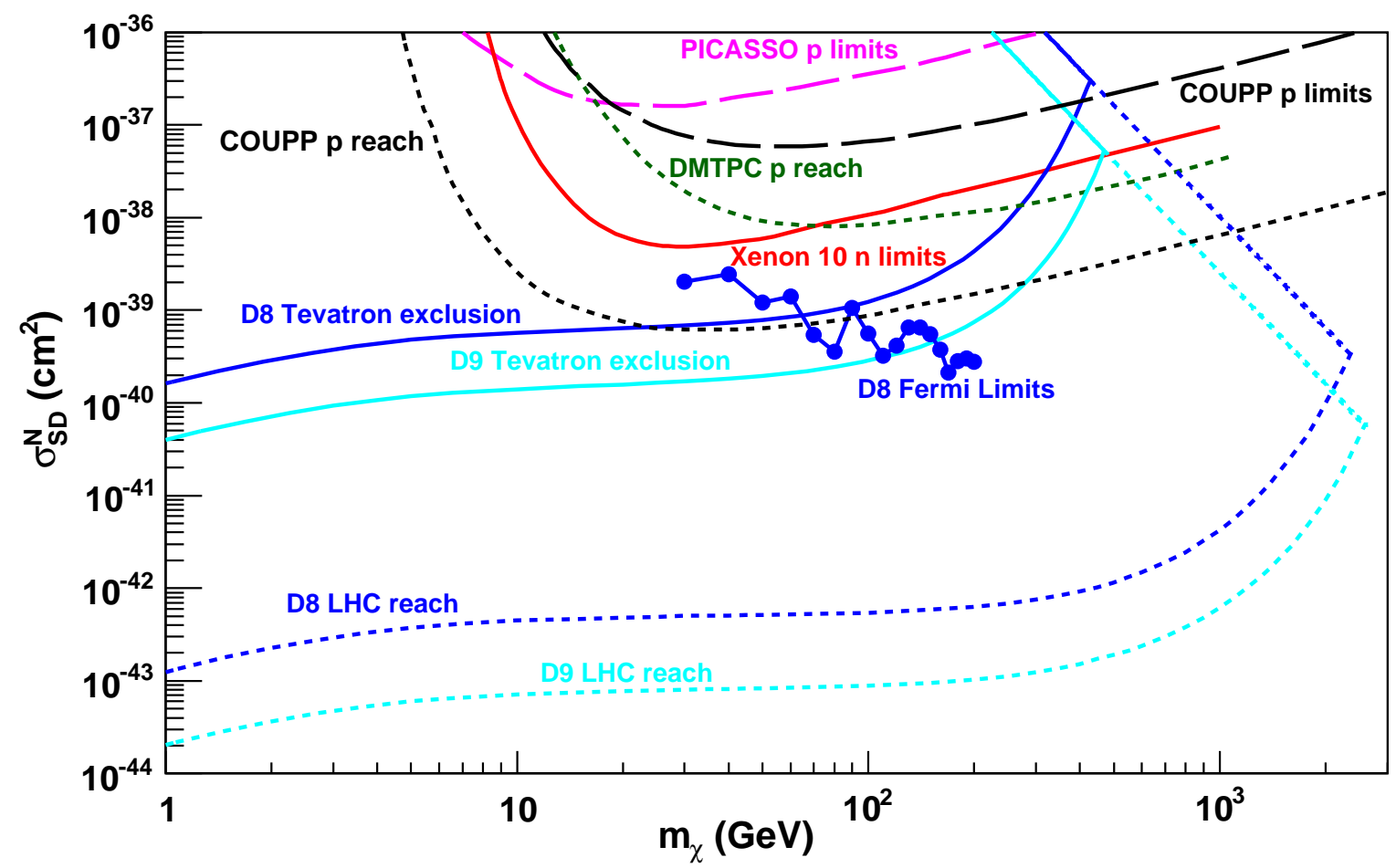

FIG. 3: Graph of the parameter space for spin-dependent direct detection of WIMPs, in terms of the WIMP mass and cross section for scattering on a proton or neutron, including current bounds from direct detection, Tevatron data, and the Fermi line search (solid lines, as labelled), and projected lines for direct detection experiments and the LHC (dashed lines, as labelled). Fermi bounds on Majorana fermion with M5 interaction are obtained by improving the D8 bounds for the Dirac fermion by a factor of 2 .

(MiDM) [36], in its electromagnetic (as opposed to dark photon) incarnation explains the DAMA signal. In this model the WIMP $\chi$ is a Majorana fermion which scatters inelastically into a slightly heavier state $\chi^{*}$ through a flavor-changing magnetic dipole moment,

$$
M \bar{\chi} \sigma^{\mu \nu} \chi^{*} F_{\mu \nu}+H . c .
$$

This operator also induces a line signal when two $\chi$ (or two $\chi^{*}$ ) annihilate into $\gamma \gamma$ by exchanging a $\chi^{*}(\chi)$. The regions correspond to $90 \%$ and $99 \%$ CL consistent parameter spaces in $m_{\chi}$ and $M$ (as found in [36]), mapped into the parameter space of indirect detection under the assumption that the dark matter halo is composed entirely of $\chi$ or $\chi^{*}$. The Fermi line search is already providing interesting constraints on the model parameter space. 

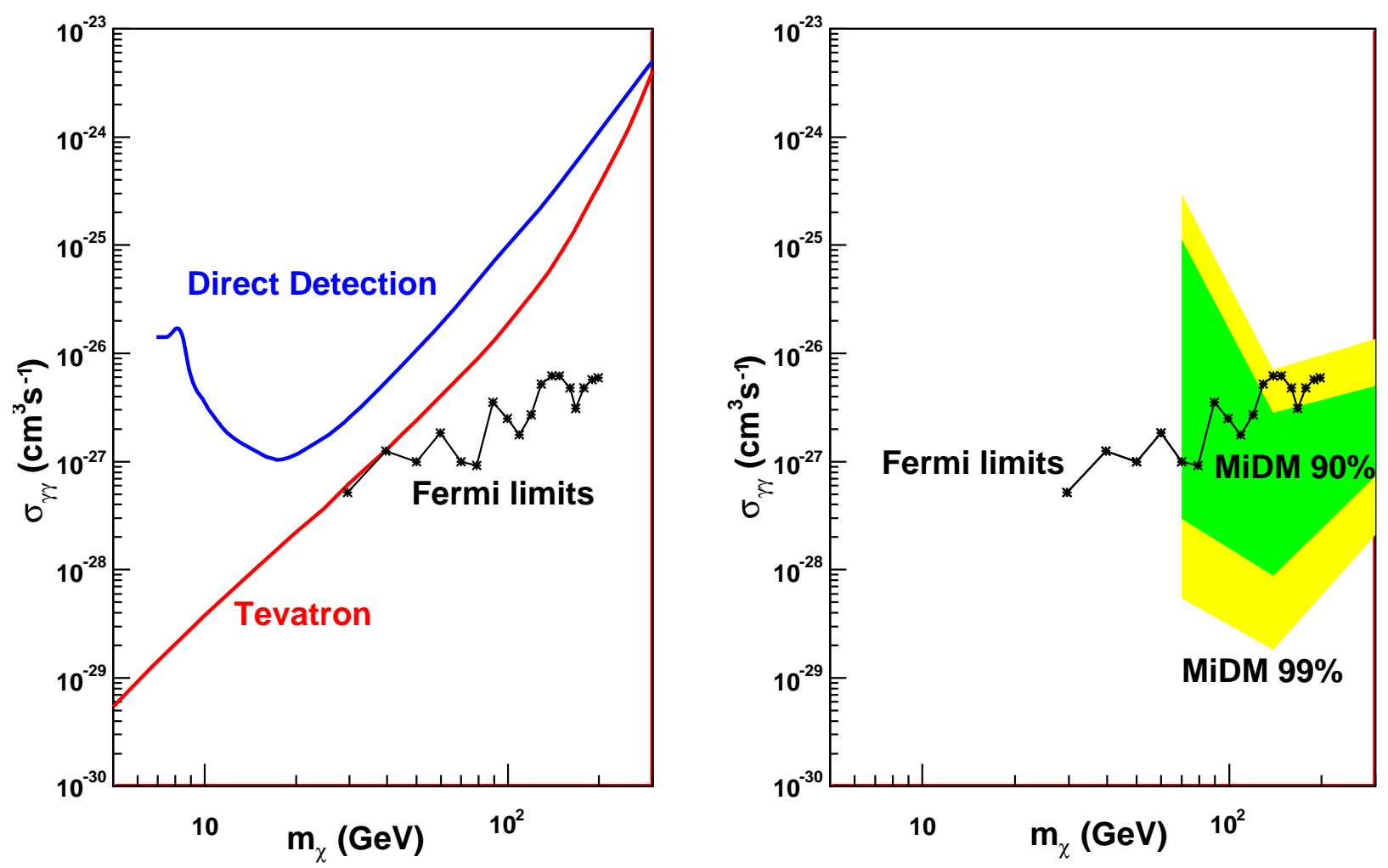

FIG. 4: On the left, a graph of the parameter space for indirect detection of gamma ray lines for a Majorana WIMP interacting primarily through operator M6, in terms of the WIMP mass and cross section annihilation into $\gamma \gamma$. Shown are the Fermi line search bounds, bounds from Tevatron data, and the direct detection experiments (solid lines, as labelled). On the right, a comparison between the Fermi line search bounds and the MiDM [36] model parameter space. The shaded regions correspond to the $90 \%$ (99\%) fits of the MiDM model to explain the DAMA signal .

Effective theories have the potential to be powerful descriptions of WIMP interactions. In this work, we have explored how the bounds from gamma ray lines map into constraints on the nature of these interactions, and seen how these constraints are an interesting, complementary picture to those provided by direct detection and colliders. All together, they provide a multi-faceted probe of the nature of dark matter. 


\section{Acknowledgements}

The authors are grateful for conversations with E. Do Couto E Silva and S. Murgia. T. Tait acknowledges the hospitality of the SLAC theory group, where part of this work was completed. This research is supported in part by NSF Grants No. PHY-0653656 and PHY-0709742.

[1] R. Bernabei et al., arXiv:1002.1028 [astro-ph.GA].

[2] J. L. Feng, J. Kumar and L. E. Strigari, Phys. Lett. B 670, 37 (2008) arXiv:0806.3746 [hep-ph]]; F. Petriello and K. M. Zurek, JHEP 0809, 047 (2008) [arXiv:0806.3989 [hep-ph]].

[3] C. E. Aalseth et al. [CoGeNT collaboration], arXiv:1002.4703 [astro-ph.CO].

[4] J. Filippini, "WIMP Hunting with the Cryogenic Dark Matter Search", Les Rencontres de Physique de la Val le dAosta (2009).

[5] E. Aprile et al. [XENON100 Collaboration], arXiv:1005.0380 [astro-ph.CO].

[6] P. Sorensen, arXiv:1007.3549 [astro-ph.IM].

[7] A. L. Fitzpatrick, D. Hooper and K. M. Zurek, arXiv:1003.0014 [hep-ph]; D. Feldman, Z. Liu and P. Nath, arXiv:1003.0437 [hep-ph].

[8] J. Kopp, T. Schwetz and J. Zupan, JCAP 1002, 014 (2010) arXiv:0912.4264 [hep-ph]].

[9] E. Kuflik, A. Pierce and K. M. Zurek, arXiv:1003.0682 [hep-ph].

[10] S. Andreas, C. Arina, T. Hambye, F. S. Ling and M. H. G. Tytgat, arXiv:1003.2595 [hep-ph]; K. J. Bae, H. D. Kim and S. Shin, arXiv:1005.5131 [hep-ph].

[11] S. Chang, J. Liu, A. Pierce, N. Weiner and I. Yavin, arXiv:1004.0697 [hep-ph].

[12] R. Essig, J. Kaplan, P. Schuster and N. Toro, arXiv:1004.0691 [hep-ph]; P. W. Graham, R. Harnik, S. Rajendran and P. Saraswat, arXiv:1004.0937 [hep-ph]; J. M. Cline, A. R. Frey and F. Chen, and arXiv:1008.1784 [hep-ph].

[13] H. An, S. L. Chen, R. N. Mohapatra, S. Nussinov and Y. Zhang, Phys. Rev. D 82, 023533 (2010) [arXiv:1004.3296 [hep-ph]].

[14] V. Barger, M. McCaskey and G. Shaughnessy, arXiv:1005.3328 [hep-ph].

[15] D. Hooper, J. I. Collar, J. Hall and D. McKinsey, arXiv:1007.1005 [hep-ph].

[16] A. Birkedal, K. Matchev and M. Perelstein, Phys. Rev. D 70, 077701 (2004) 
arXiv:hep-ph/0403004]; P. Konar, K. Kong, K. T. Matchev and M. Perelstein, New J. Phys. 11, 105004 (2009) arXiv:0902.2000 [hep-ph]]; J. L. Feng, S. Su and F. Takayama, Phys. Rev. Lett. 96, 151802 (2006) arXiv:hep-ph/0503117.

[17] Q. H. Cao, C. R. Chen, C. S. Li and H. Zhang, arXiv:0912.4511 [hep-ph].

[18] M. Beltran, D. Hooper, E. W. Kolb, Z. A. C. Krusberg and T. M. P. Tait, arXiv:1002.4137 [hep-ph].

[19] J. Goodman, M. Ibe, A. Rajaraman, W. Shepherd, T. M. P. Tait and H. B. Yu, arXiv:1005.1286 [hep-ph]; J. Goodman, M. Ibe, A. Rajaraman, W. Shepherd, T. M. P. Tait and H. B. Yu, arXiv:1008.1783 [hep-ph].

[20] Y. Bai, P. J. Fox and R. Harnik, arXiv:1005.3797 [hep-ph].

[21] G. D. Mack, T. D. Jacques, J. F. Beacom, N. F. Bell and H. Yuksel, Phys. Rev. D 78, 063542 (2008) arXiv:0803.0157 [astro-ph]].

[22] L. Bergstrom and P. Ullio, Nucl. Phys. B 504, 27 (1997) arXiv:hep-ph/9706232; Z. Bern, P. Gondolo and M. Perelstein, Phys. Lett. B 411, 86 (1997) arXiv:hep-ph/9706538; P. Ullio and L. Bergstrom, Phys. Rev. D 57, 1962 (1998) arXiv:hep-ph/9707333]; L. Bergstrom, P. Ullio and J. H. Buckley, Astropart. Phys. 9, 137 (1998) arXiv:astro-ph/9712318; F. Boudjema, A. Semenov and D. Temes, Phys. Rev. D 72, 055024 (2005) arXiv:hep-ph/0507127.

[23] L. Bergstrom, T. Bringmann, M. Eriksson et al., JCAP 0504, 004 (2005). hep-ph/0412001]; G. Bertone, C. B. Jackson, G. Shaughnessy, T. M. P. Tait and A. Vallinotto, Phys. Rev. D 80 (2009) 023512 arXiv:0904.1442 [astro-ph.HE]]; C. B. Jackson, G. Servant, G. Shaughnessy, T. M. P. Tait and M. Taoso, JCAP 1004, 004 (2010) [arXiv:0912.0004 [hep-ph]].

[24] E. Dudas, Y. Mambrini, S. Pokorski and A. Romagnoni, arXiv:0904.1745 [hep-ph]; Y. Mambrini, JCAP 0912, 005 (2009) arXiv:0907.2918 [hep-ph]]; C. Arina, T. Hambye, A. Ibarra and C. Weniger, JCAP 1003, 024 (2010) [arXiv:0912.4496 [hep-ph]].

[25] M. Perelstein and A. Spray, Phys. Rev. D 75 (2007) 083519 arXiv:hep-ph/0610357; M. Gustafsson, E. Lundstrom, L. Bergstrom and J. Edsjo, Phys. Rev. Lett. 99 (2007) 041301 arXiv:astro-ph/0703512.

[26] M. Beltran, D. Hooper, E. W. Kolb and Z. C. Krusberg, Phys. Rev. D 80, 043509 (2009) arXiv:0808.3384 [hep-ph]]; J. Fan, M. Reece and L. T. Wang, arXiv:1008.1591 [hep-ph].

[27] W. Shepherd, T. M. P. Tait and G. Zaharijas, Phys. Rev. D 79, 055022 (2009) arXiv:0901.2125 [hep-ph]]. 
[28] M. Cirelli, M. Kadastik, M. Raidal and A. Strumia, Nucl. Phys. B 813, 1 (2009) arXiv:0809.2409 [hep-ph]]; C. Arina and M. H. G. Tytgat, arXiv:1007.2765 [astro-ph.CO]; M. Perelstein and B. Shakya, arXiv:1007.0018 [astro-ph.HE]; A. Badin, G. K. Yeghiyan and A. A. Petrov, arXiv:0909.5219 [hep-ph].

[29] A. J. Buras, P. Gambino, M. Gorbahn, S. Jager and L. Silvestrini, Phys. Lett. B 500, 161 (2001) arXiv:hep-ph/0007085.

[30] K. Sigurdson, M. Doran, A. Kurylov, R. R. Caldwell and M. Kamionkowski, Phys. Rev. D 70, 083501 (2004) [Erratum-ibid. D 73, 089903 (2006)] [arXiv:astro-ph/0406355]; S. Profumo and K. Sigurdson, Phys. Rev. D 75, 023521 (2007) arXiv:astro-ph/0611129].

[31] J. Bagnasco, M. Dine and S. D. Thomas, Phys. Lett. B 320, 99 (1994) arXiv:hep-ph/9310290].

[32] M. Pospelov and T. ter Veldhuis, Phys. Lett. B 480, 181 (2000) arXiv:hep-ph/0003010.

[33] S. Gardner, Phys. Rev. D 79, 055007 (2009) [arXiv:0811.0967 [hep-ph]].

[34] W. S. Cho, J. H. Huh, I. W. Kim, J. E. Kim and B. Kyae, Phys. Lett. B 687, 6 (2010) arXiv:1001.0579 [hep-ph]].

[35] E. Masso, S. Mohanty and S. Rao, Phys. Rev. D 80, 036009 (2009) [arXiv:0906.1979 [hep-ph]].

[36] S. Chang, N. Weiner and I. Yavin, arXiv:1007.4200 [hep-ph].

[37] V. Barger, W. Y. Keung and D. Marfatia, arXiv:1007.4345 [hep-ph].

[38] T. Banks, J. F. Fortin and S. Thomas, arXiv:1007.5515 [hep-ph].

[39] A. L. Fitzpatrick and K. M. Zurek, arXiv:1007.5325 [hep-ph].

[40] L. D. Landau, Dokl. Akad. Nawk., USSR 60, 207 (1948); C. N. Yang, Phys. Rev. 77, 242 (1950).

[41] T. Hahn, Comput. Phys. Commun. 140, 418 (2001) arXiv:hep-ph/0012260.

[42] T. Hahn and M. Perez-Victoria, Comput. Phys. Commun. 118, 153 (1999) arXiv:hep-ph/9807565.

[43] G. Passarino and M. J. G. Veltman, Nucl. Phys. B 160, 151 (1979).

[44] A. R. Pullen, R. R. Chary and M. Kamionkowski, Phys. Rev. D 76, 063006 (2007) arXiv:astro-ph/0610295.

[45] A. A. Abdo et al., Phys. Rev. Lett. 104, 091302 (2010) arXiv:1001.4836 [astro-ph.HE]].

[46] J. F. Navarro, C. S. Frenk and S. D. M. White, Astrophys. J. 462, 563 (1996) arXiv:astro-ph/9508025.

[47] A. W. Graham, D. Merritt, B. Moore, J. Diemand and B. Terzic, Astron. J. 132, 2685 (2006) 


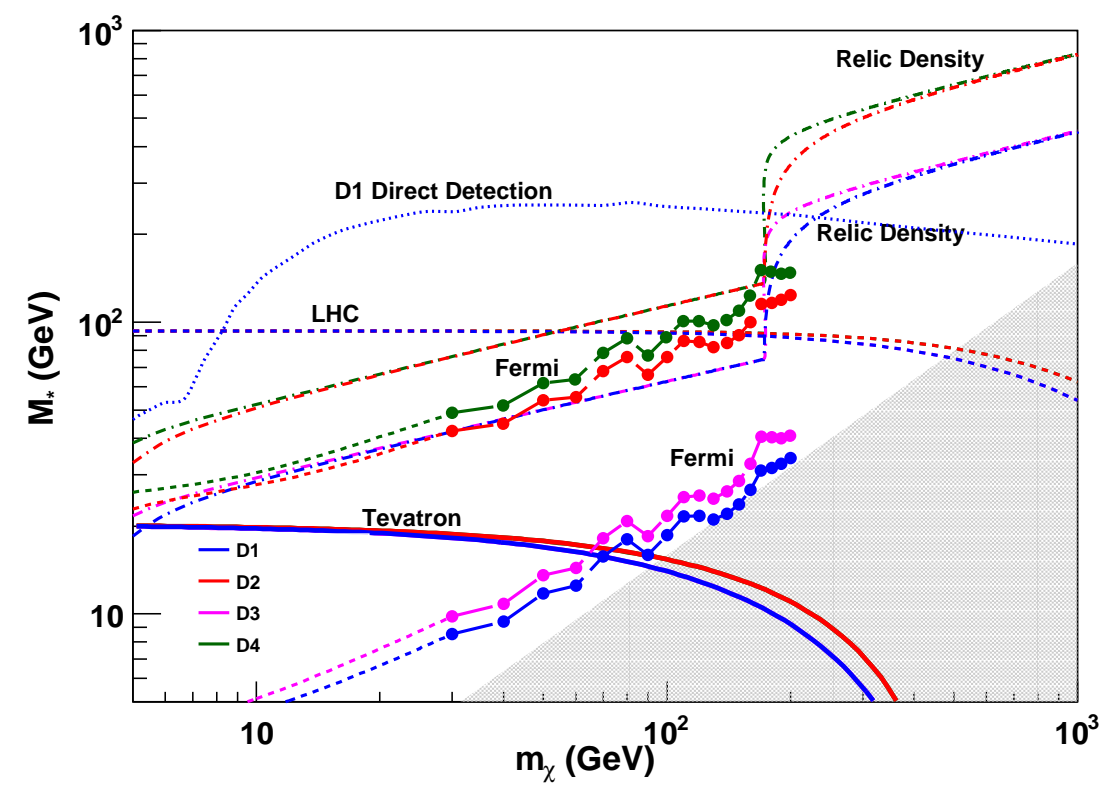

FIG. 5: The lower limits from current Fermi line searches [45] (long dashed lines with data points shown) and an estimate of the reach of future searches at lower energies (short dashed curve) on the suppression scale of new physics $M_{*}$ leading to interactions with the SM for the Dirac WIMP operators D1-D4. Note that the constraints on D1 and D3 are significantly weaker than the others, because these operators lead to cross sections which are velocity-suppressed. For comparison we also show the current bounds from Tevatron and future reach of LHC [19] (solid and short dashed curves, respectively), as well as the value of $M_{*}$ leading to the correct thermal relic abundance in the absence of other interactions (dash-dotted curves).

[arXiv:astro-ph/0509417].

[48] J. N. Bahcall and R. M. Soneira, Astrophys. J. Suppl. 44, 73 (1980).

[49] Z. Ahmed et al. [The CDMS-II Collaboration], arXiv:0912.3592 [astro-ph.CO]; J. Angle et al. [XENON Collaboration], Phys. Rev. Lett. 100, 021303 (2008) arXiv:0706.0039 [astroph]]; D. S. Akerib et al., Nucl. Instrum. Meth. A 559, 411 (2006); E. Aprile, L. Baudis and f. t. X. Collaboration, PoS IDM2008, 018 (2008) arXiv:0902.4253 [astro-ph.IM]].

[50] S. Archambault et al., Phys. Lett. B 682, 185 (2009) arXiv:0907.0307 [hep-ex]]; H. S. Lee et al. [KIMS Collaboration], Phys. Rev. Lett. 99, 091301 (2007) arXiv:0704.0423 [astro-ph]]; G. Sciolla et al., J. Phys. Conf. Ser. 179, 012009 (2009) arXiv:0903.3895 [astro-ph.IM]]; E. Behnke et al., arXiv:1008.3518 [astro-ph.CO]. 


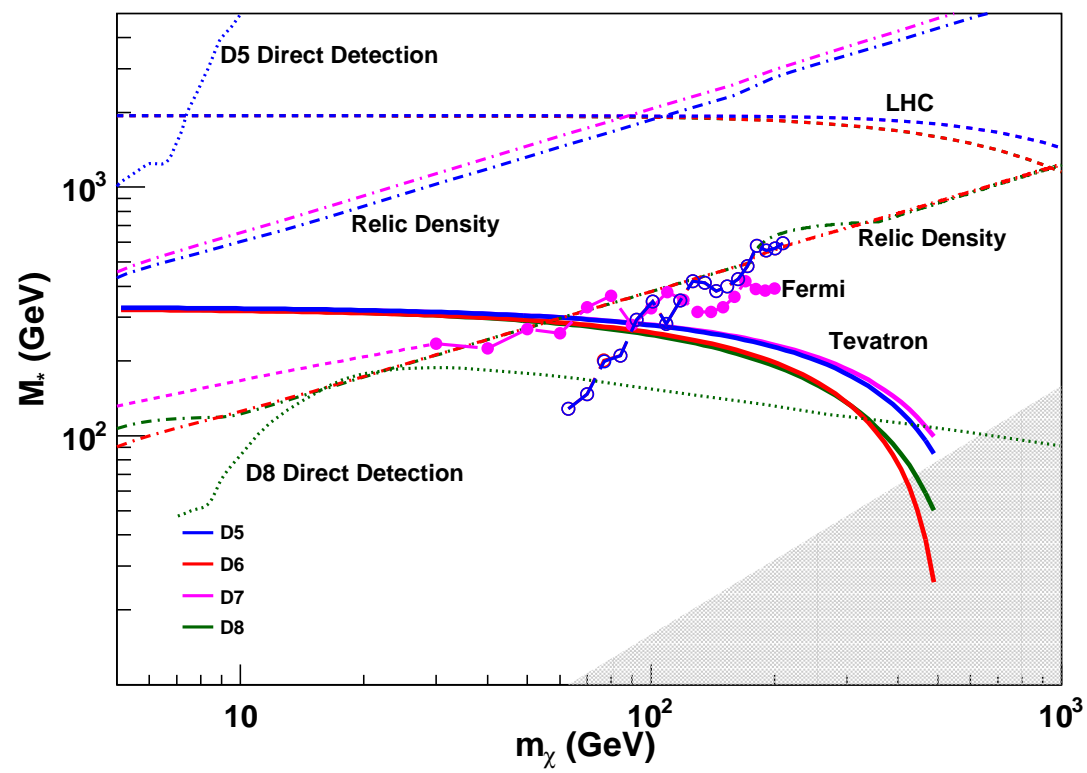

FIG. 6: Same as figure5, but for the Dirac WIMP operators D5-D8. Constraints for D5 and D6 arise from the $\gamma Z$ annihilation process, and thus there are no effective line search bounds for masses $m_{\chi} \lesssim 50 \mathrm{GeV}$

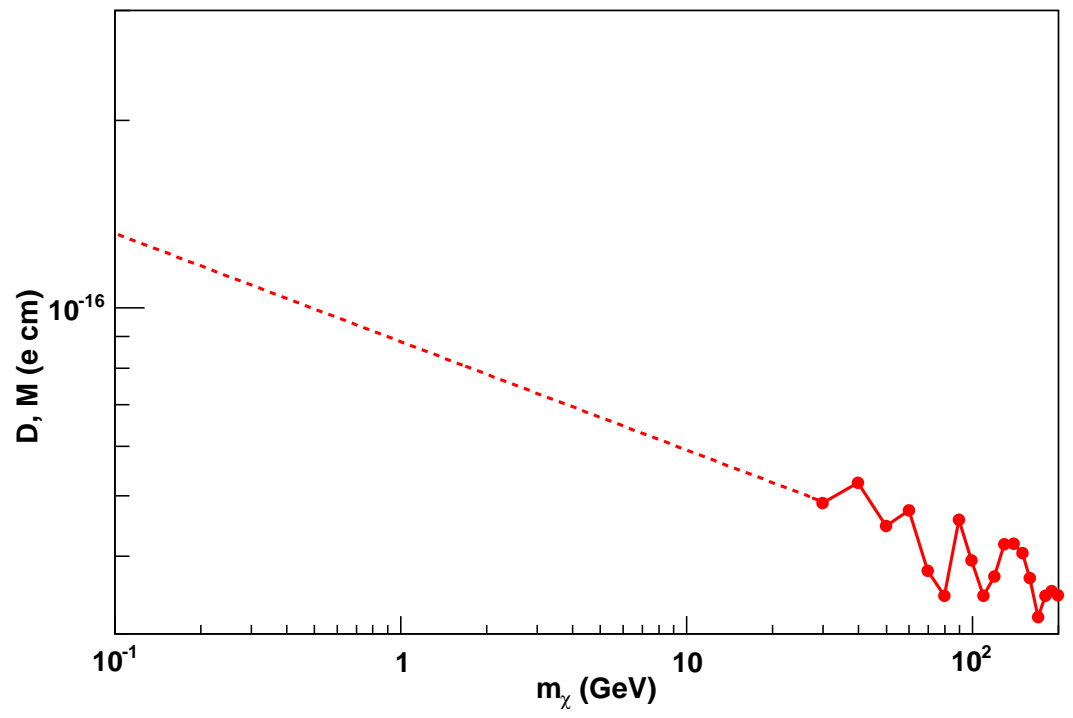

FIG. 7: Experimental upper bounds on dipole moments of dark matter (operators D15 and D16) derived from the spectral line searches by FERMI [45] (solid lines). The dashed curve is our estimate of the reach of a future Fermi line search covering the illustrated lower energy range. 


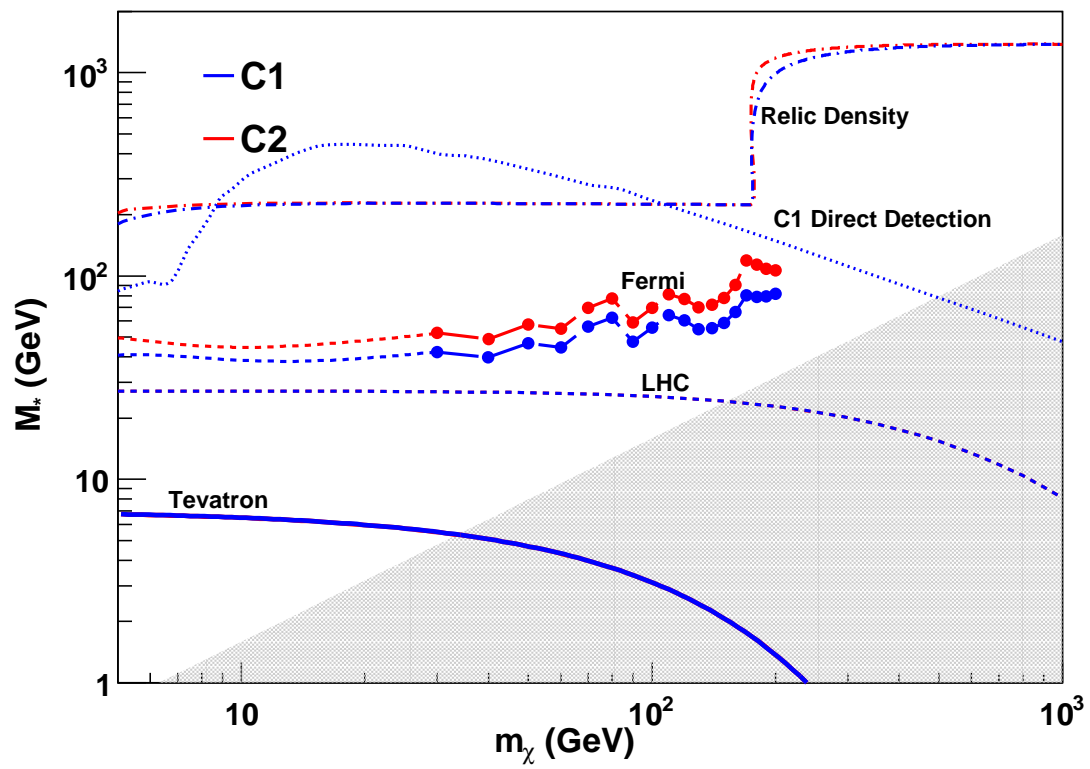

FIG. 8: Same as figure5, but with constraints shown for the complex scalar WIMP interacting with the SM through operators $\mathrm{C} 1$ and $\mathrm{C} 2$.

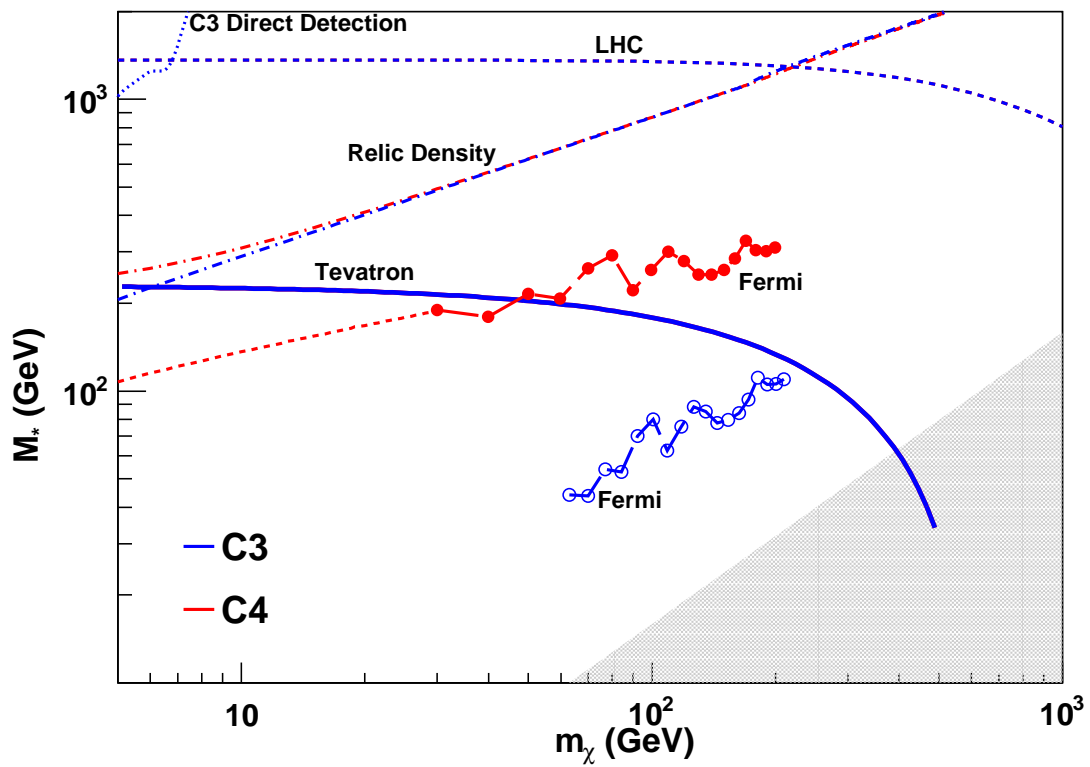

FIG. 9: Same as figure5, but with constraints shown for the complex scalar WIMP interacting through operators $\mathrm{C} 3$ and C4. Operator C3 is only constrained by the $\gamma Z$ annihilation process, and thus there are no effective line search bounds for masses $m_{\chi} \lesssim 50 \mathrm{GeV}$. 